\title{
Salivary Biomarkers May Be Useful to Assess Stress State in Patients with Lung Cancer Undergoing Chemotherapy
}

\author{
Maiko Kitajima $^{1}$, Yuka Noto ${ }^{1}$, Umi Kudo ${ }^{1}$, Mayumi Urushizaka ${ }^{1}$, Toshiko Tomisawa ${ }^{1}$, Chieko Itaki $^{1}$, \\ Kumiko Kawasaki ${ }^{1}$, Hideaki Yamabe ${ }^{1}$, Akihito Hayashi ${ }^{2}$, Shingo Takanashi $^{2}$ \\ ${ }^{1}$ Hirosaki University Graduate School of Health Sciences, Hirosaki, Japan; ${ }^{2}$ Department of Respiratory Medicine, Hirosaki Univer- \\ sity Hospital, Hirosaki, Japan. \\ Email: kitajima@cc.hirosaki-u.ac.jp
}

Received August 24 ${ }^{\text {th }}, 2011$; revised October $5^{\text {th }}, 2011$; accepted October $24^{\text {th }}, 2011$.

\begin{abstract}
Objective: As it is important for medical staff to understand the stress state of patients with cancer, this study investigated the stress state in patients with lung cancer undergoing chemotherapy using salivary biomarkers and questionnaires. Materials and Methods: The subjects included 19 men and 9 women, with a mean age of $66.1 \pm 7.1$ years who received chemotherapy for lung cancer. Salivary samples of the participants were collected before and the 7 day after chemotherapy. Simultaneously, they completed the POMS (profile of mood states) and QOL (quality of life) questionnaires. The salivary cortisol and secretory IgA as stress markers were measured by enzyme immunoassay. Results: The salivary secretory IgA level was significantly higher at the 7th day after chemotherapy than before chemotherapy $(p<$ $0.05)$, although the salivary cortisol level did not change. The tension-anxiety score, which is one of POMS subclasses, and total mood disturbance score were lower at day 7 of chemotherapy than before chemotherapy $(p<0.05)$, although no correlations were observed between POMS scores and salivary markers. However, a negative correlation was observed between the salivary cortisol level and the QOL full score $(r=-0.411, p<0.05)$. Conclusions: Measurement of salivary stress markers is noninvasive and may be useful for assessing the stress state of the patients.
\end{abstract}

Keywords: Lung Cancer, Chemotherapy, Secretory-IgA, Saliva, Stress Marker

\section{Introduction}

Lung cancer is the leading cause of cancer-related deaths in Japan since 1998 [1]. The incidence of this disease is increasing yearly due to smoking and the increase in elderly people. Patients who suffer from lung cancer are usually treated with surgery, chemotherapy and/or radiotherapy. Recently, chemotherapy has been shown to be very effective for patients with small cell lung cancer. Therefore, medical staff should administer chemotherapy safely and correctly to ensure optimum outcomes for these patients. Moreover, they should try to reduce the level of pain experienced by the patient, maintain the physical condition of the patient, minimize the toxic side effects of chemotherapy, and reduce physical and emotional stresses.

In 1950, Selye described the General Adaptation Syndrome for the response of the body to stress [2,3]. Lazarus described stress as a process involving complex in- teractions between the environment and a living body [4]. Patients who have cancer undergo numerous physical and emotional stresses. The stresses suffered by cancer patients who are undergoing chemotherapy are related to the diagnosis of cancer, notification of malignancy, fear of relapse, symptoms of cancer, changes in life-style, and the toxic side effects of chemotherapy. Excessive stress may debilitate the immune system, resulting in complications of cancer and exacerbating the cancer itself.

This study investigated stress assessment in patients with lung cancer who are receiving chemotherapy by examining biomarkers in the saliva and using the Profile of Mood States (POMS) and Quality of Life (QOL) questionnaires. The correlation between two stress markers in the saliva and the QOL index were also examined. Generally, when the degree of their stress is known, the questionnaire is used like POMS. However, completion of questionnaires can be a large burden for aged cancer 
patients undergoing treatment. Thus, an alternative method by collecting their saliva to measure stress levels may be less of a burden. The stress state of the patients was thus investigated by determining the correlation of both assessment scales.

\section{Materials and Methods}

\subsection{Subjects}

This study included lung cancer patients who received chemotherapy at Hirosaki University Hospital, Japan. This study was approved by the Committee for Medical Ethics of Hirosaki University, and the patients gave informed consent. There were 28 patients (19 men and 9 women) who participated in this study. The average age was $66.1 \pm 7.1$ years. Saliva samples were collected from all patients. Six patients were unable to complete Functional Assessment of Cancer Therapy (FACT) questionnaires because they were too tired at that time. The patient's characteristics are shown in Table 1. No patient had an operation for lung cancer or any complication, such as peptic ulcer or a cerebral accident during the study. From the beginning of chemotherapy to day 7 after chemotherapy, side effects including malaise and fever appeared in $50 \%$ of the patients.

\subsection{Examination of Salivary Biomarkers and Questionnaires}

The subjects were required to abstain from eating and drinking anything except water and from brushing their teeth $1 \mathrm{~h}$ before obtaining saliva samples. Samples were obtained twice from each patient between 14:00 h and 18:00 h, to minimize any circadian rhythm effects. Emotional status was assessed using the Japanese version of POMS, and QOL was assessed using the Functional Assessment of Cancer Therapy-Lung (FACT-L). The patients completed these questionnaires after collecting of the salivary samples before and at day 7 of chemotherapy (Figure 1). When it was difficult for the patients to reply to both questionnaires, they were requested to complete the POMS only.

\subsection{Assaying Biomarkers in the Saliva of Patients with Lung Cancer}

Salivary samples were obtained from the patients using Salivette (Sarstedt AG \& Co., Nümbrecht, Germany) with a straw directly. Salivette easily collected saliva by allowing the subject to chew on a roll-shaped saliva collector after initially swallowing the saliva present in the mouth. The samples were centrifuged for $5 \mathrm{~min}$ at $3000 \mathrm{rpm}$, and the supernatants were frozen at $-80^{\circ} \mathrm{C}$ until use. Salivary samples were analyzed for the levels of the biomarkers
Table 1. The subject characteristics $(n=28)$.

\begin{tabular}{ll}
\hline \multicolumn{1}{c}{ Characteristics } & Number of subjects \\
\hline Gender & 19 \\
Male & 9 \\
Female & \\
Histology & 16 \\
Adenocarcioma & 3 \\
Squamous cell arcinoma & 9 \\
Small cell carcinoma & \\
Disease stage (Adenocarcioma Squamous \\
cell arcinoma) \\
Stage IIIA
\end{tabular}

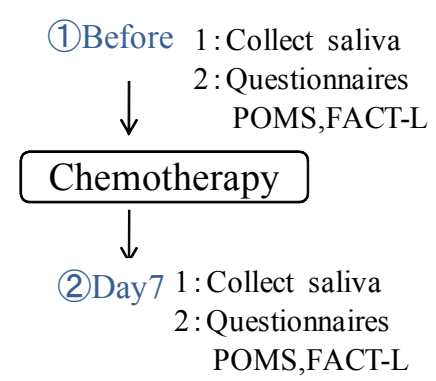

Figure 1. The protocol of this research. The participants completed the questionnaires of POMS and FACT-L after the collection of salivary samples, which were performed twice, before and the 7th day of chemotherapy. 
cortisol and secretory IgA (s-IgA). Salivary cortisol was estimated using a salivary cortisol enzyme immunoassay kit (Salimetrics, State College, PA, USA) and salivary s-IgA was estimated using a salivary s-IgA indirect enzyme immunoassay kit (Salimetrics) according to the manufacturer's instructions. These are competitive immunoassay kits specifically designed for the quantitative measurement of salivary cortisol and s-IgA.

\subsection{Subjective Evaluation Index of Stress}

The POMS questionnaire, which was developed and validated by McNair et al. in the United States [5], is a self-administered instrument that consists of 65 items designed to identify and assess transient mood states. It measures six mood or affective states: tension-anxiety, depression-dejection, anger-hostility, vigor, fatigue and confusion. It can also calculate the total mood disturbance (TMB) score which expresses negative mood. Yokoyama translated the POMS questionnaire into Japanese and validated it [6]. The shortened version of the POMS questionnaire consists of 30 items and can be completed in only 10 minutes. The changes in feelings before and after an intervention can be measured. The shortened version generates the same results as the original POMS questionnaire. A standardization score is computed from the measurements and is compared with the average according to age.

\subsection{QOL of Patients with Lung Cancer}

QOL is significantly affected by the occurrence of side effects, such as nausea/vomiting, loss of appetite, and diarrhea [7]. However, the relationship between the degree of stress and QOL has yet to be precisely defined.

The Functional Assessment of Cancer Therapy-General (FACT-G) scale was developed and validated by Cella et al. [8]. In Japan, Fumimoto et al. demonstrated the clinical validity of the cross-cultural validation of the Japanese FACT-G scale [9]. FACT-G questionnaires consist of four domains: 1) physical well-being; 2) social/family well-being; 3) emotional well-being; and 4) functional well-being. For patients with lung cancer, the FACT-L questionnaire is used in combination with the Lung Cancer Subscale. The FACT-L (version 3) questionnaire is a 44-item self-reporting instrument that measures a multidimensional QOL $[10,11]$.

\subsection{Statistical Analysis}

All data are expressed as the mean \pm standard deviation and analyzed using SPSS 11.5 software. Results were compared using Wilcoxon signed rank test, Mann-Whitney and Spearman's rank correlation coefficient. $P<0.05$ was considered to be statistically significant.

\section{Results}

\subsection{Transition of Salivary Biomarkers}

For the 28 patients, the mean cortisol level before and after chemotherapy was $0.26 \pm 0.41$ and $0.37 \pm 0.47$ $\mu \mathrm{g} / \mathrm{dl}$, respectively. The mean s-IgA level before and after chemotherapy was $190.6 \pm 164.3$ and $288.6 \pm 292.0$ $\mu \mathrm{g} / \mathrm{ml}$, respectively. The salivary s-IgA level was significantly higher at day 7 of chemotherapy than before chemotherapy $(p<0.05)$. There was no significant difference between the salivary cortisol level before and after chemotherapy (Figure 2). We did not observe any relation with pathological type of lung cancer, disease stage and gender in the levels of salivary markers.

\subsection{POMS and QOL Scores for Patients}

The POMS scores for the patients are presented in Table 2. The POMS subscales of tension-anxiety and TMD scores were lower at day 7 of chemotherapy than before chemotherapy $(p<0.05)$. No significant differences were found between before chemotherapy and at day 7 of chemotherapy in the FACT-L questionnaire scores. We did not observe any relation with pathological type of lung cancer, disease stage and gender in the POMS and QOL scores.

\subsection{Relationship between Salivary Biomarkers and QOL Scores}

The A negative correlation was observed between the salivary cortisol levels and the QOL full score $(\mathrm{r}=-0.403$, $\mathrm{p}<0.05$ ), which means that the higher the salivary cortisol levels, the lower the QOL full score (Figure 3). No

Transition of salivary biomarkers $(\mathrm{n}=28)$

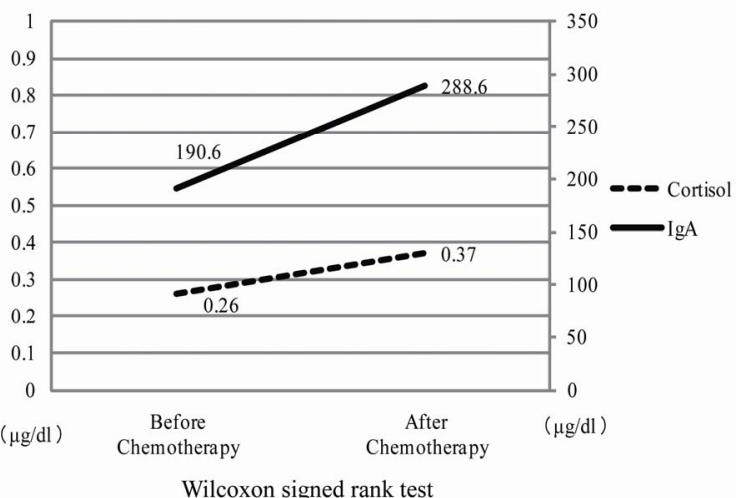

Figure 2. Transition of salivary biomarkers $(n=28)$. The salivary s-IgA level was significantly higher at day 7 of chemotherapy than before chemotherapy $(p<0.05)$. There was no significant difference between the salivary cortisol level before and after chemotherapy. 
Table 2. The progress of Questionnaires' scores and stress marker level in saliva $(n=28)$. The POMS subscales of tension-anxiety and TMD score were significantly lower at the $7^{\text {th }}$ day of chemotherapy than before chemotherapy $(p<0.05)$. No significant differences were found between before chemotherapy and at day 7 of chemotherapy in the FACT-L questionnaire scores.

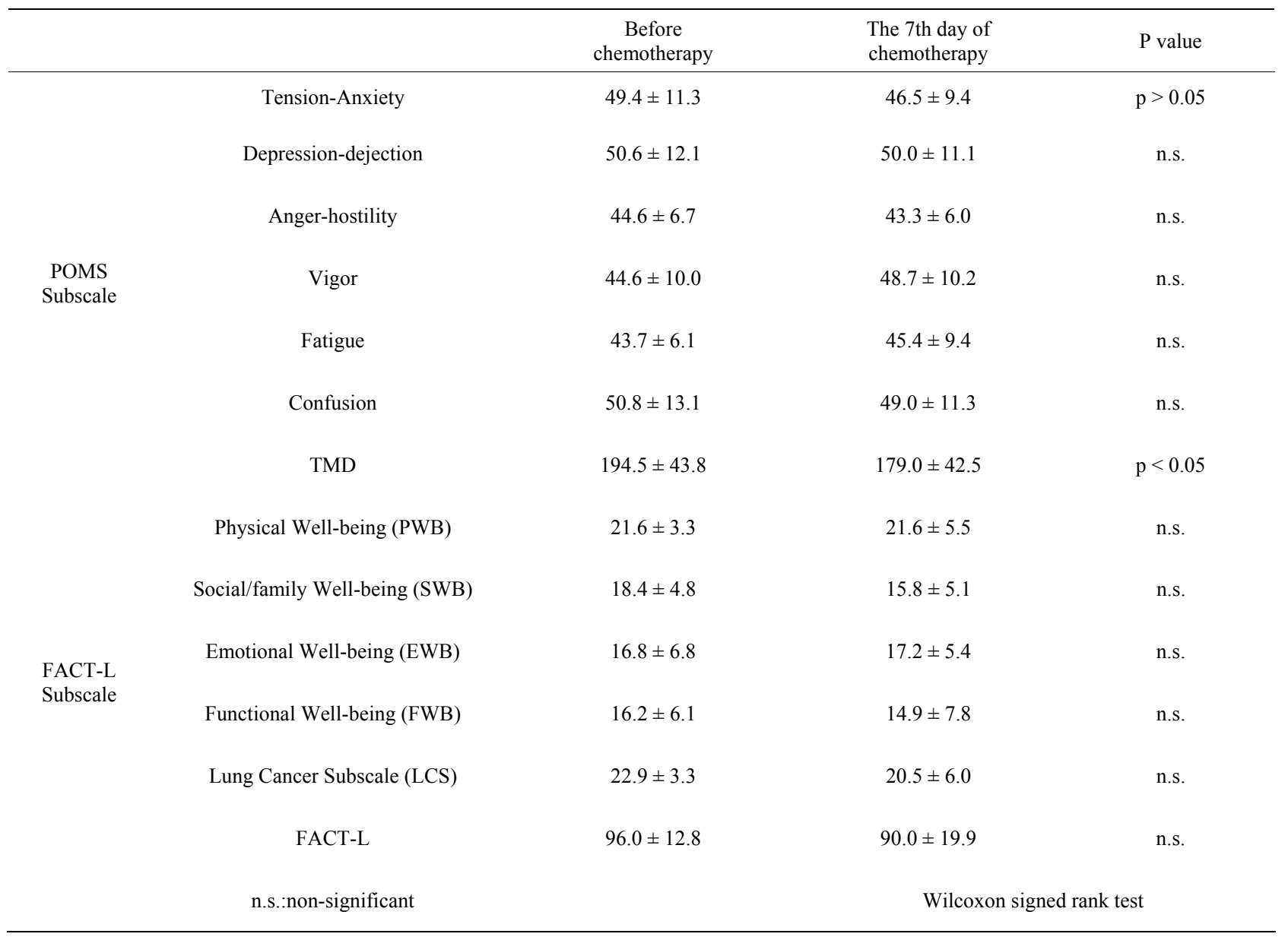

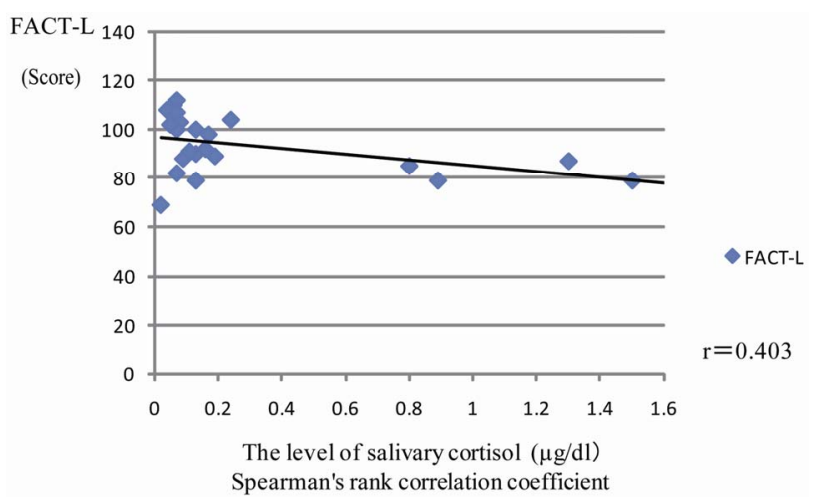

Figure 3. Correlation of the salivary cortisol levels and FACT-L score $(n=25)$. A negative correlation was observed between the salivary cortisol levels and the FACT-L full score $(r=-0.403, P<0.05)$.

significant relationship was noted between the salivary s-IgA levels and the any QOL scores.

\section{Discussion}

Patients with cancer who are receiving chemotherapy are usually exposed to various kinds of stresses. Excessive stress may worsen the physical and mental conditions of these patients and exacerbate the cancer itself. In this study, we investigated stress assessment in patients with lung cancer who were receiving chemotherapy, by assaying biomarkers in the saliva and administering the POMS and QOL questionnaires to determine correlations between the levels of two stress markers in the saliva and the POMS or QOL index.

Numerous physiologic measures have been evaluated for their roles in the stress response, e.g., pulse rate, blood pressure, sweating, and the cortisol, epinephrine, and norepinephrine levels [12]. One of the most common objective measures of stress involves the assaying of neuroendocrine markers. The cortisol level in the blood is known to increase under mental stress or pain [13], 
while the salivary cortisol level increases with psychological stress [13-15] and correlates with the level of serum cortisol [15]. Saliva can be easily collected by allowing patients to chew on a roll-shaped saliva collector after initially swallowing the saliva present in the mouth. Since salivary measurements are non-invasive, salivary biomarkers may be useful clinical indicators of mental stress.

S-IgA is immunoglobulin A contained in secretions like saliva. About $95 \%$ of s-IgA is produced by immune cells in salivary glands rather than lead from serum $[16,17]$. S-IgA is also thought to be a remarkable stress marker. It is reported that the immediate stress increases s-lgA secretion rapidly, and the delayed stress decreases s-lgA secretion several days after stress [18]. Graham et al. reported that more frequent episodes of anxiety significantly lowers s-IgA [19]. S-IgA levels have been shown to fall by physical stress such as exercise training [20].

In this study, the levels of cortisol and s-IgA in the saliva of cancer patients were measured before and during chemotherapy. As a result, the salivary level of s-IgA was significantly higher at day 7 of chemotherapy than before chemotherapy. Although we found no significant changes in salivary cortisol levels before or after chemotherapy, Kajdaniuk et al. reported increased cortisol levels, which might be the result of stress, in blood samples of women with breast cancer before the first cycle of supplementary chemotherapy [21].

We also found that the POMS subscales of tensionanxiety and the TMD score were lower at day 7 of chemotherapy than before chemotherapy. A high score of tension-anxiety implies that the patients have become more tense. The TMD score is the score which takes off the vigor score from the total scores of five subscales. A high TMD indicates that the patients have a high negative mood. Since the patients in this study underwent chemotherapy for the first time, we expected a worse negative mood mainly concerned with tension due to medical treatment and this tension might be relieved at day 7 of chemotherapy (at the end of the first treatment) with the support of medical staff. Therefore, an increase in salivary s-IgA may be expected after chemotherapy.

A negative correlation was observed between the salivary cortisol levels and QOL full scores, suggesting the higher the level of salivary cortisol, the lower the full score for QOL. In a review about QOL in patients with lung cancer from 1970 to 1995 , palliation of symptoms, psychosocial interventions, and understanding of patients' feelings and concerns were reported to all contribute to improving QOL of lung cancer patients [22]. The salivary level of cortisol may reveal significant associa- tions with several psychological characteristics and serve as a negative profile marker in patients with cancer. Stress factors include age, sleeping quality, and gender. Although cortisol levels have been associated with significantly poorer sleep quality [23], all of the patients in this study reported high-quality sleep. Therefore, we thought the QOL score did not change after chemotherapy in this study.

The mean salivary cortisol levels have been reported to correlate negatively with the subscales of appraisal, belonging, and tangible social support in the Interpersonal Support Evaluation List [24]. Therefore, mental health support is essential to maintain good QOL during the total chemotherapy period. It is very important for medical staff to understand the stress state of patients with cancer and to support these patients using nursing practices such as aromatherapy, foot care, and music therapy. In that case, measurement of salivary biomarkers may be a useful method for objectively evaluating the effect of nursing practices

In this study, the POMS score decreased as salivary s-IgA increased after chemotherapy in patients with lung cancer undergoing chemotherapy. A negative relation was observed between the QOL score and the salivary cortisol level. Thus, measurement of salivary stress markers is noninvasive and may be useful for assessing the stress state of patients.

\section{Acknowledgements}

The main support for this study was provided by the medical staff of Hirosaki University Hospital, and I would like to thank all the participants, especially those patients who devoted so much time and energy to this study. This work was supported by a Grant-in-Aid for the Japanese Ministry of Education, Culture, Sports, Science and Technology Scientific Research (Young Scientists (B) 21792199).

\section{REFERENCES}

[1] Center for Cancer Control and Information Services, National Cancer Center, Japan, "Age-Specific Mortality Rate-Lung," 2009.

http://ganjoho.jp/pro/statistics/gdball.html? 1\%2\%1

[2] H. Selye and C. Fortier, "Adaptive Reaction to Stress," Psychosomatic Medicine, Vol. 12, No. 3, 1950, pp. 149157.

[3] H. Selye, "Stress and the General Adaptation Syndrome," British Medical Journal, Vol. 1, 1950, pp. 383-392. doi:10.1136/bmj.1.4667.1383

[4] R. S. Lazarus and S. Folkman, "Stress, Appraisal, and Coping," Springer Publishing, New York, 1984.

[5] D. M. McNair, M. Lorr, L. F. Droppleman, et al., "Profile 
of Mood States," Educational and Industrial Testing Service, San Diego, 1992.

[6] K. Yokoyama, S. Araki, N. Kawakami, et al., "Production of the Japanese Edition of Profile of Mood States (POMS): Assessment of Reliability and Validity," Japanese Journal of Public Health, Vol. 37, 1990, pp. 913918.

[7] S. Morita, K. Kobayashi, K. Eguchi, et al., "Influence of Clinical Parameters on Quality of Life during Chemotherapy in Patients with Advanced Non-Small Cell Lung Cancer: Application of a General Linear Model," Japanese Journal of Clinical Oncology, Vol. 33, No. 9, 2003, pp. 470-476. doi:10.1093/jjco/hyg083

[8] D. F. Cella, D. S. Tulsky, G. Gray, et al., "The Functional Assessment of Cancer Therapy Scale: Development and Validation of the General Measure," Journal of Clinical Oncology, Vol. 11, No. 3, 1993, pp. 570-579.

[9] H. Fumimoto, K. Kobayashi, C. H. Chang, et al., "Crosscultural Validation of an International Questionnaire, the General Measure of the Functional Assessment of Cancer Therapy Scale (FACT-G)," Quality of Life Research, Vol. 10, 2001, pp. 701-709. doi:10.1023/A:1013851216181

[10] D. F. Cella, A. E. Bonomi, S. R. Lloyd, et al., "Reliability and Validity of the Functional Assessment of Cancer Therapy-Lung (FACT-L) Quality of Life Instrument," Lung Cancer, Vol. 12, No. 3, 1995, pp. 199-220. doi:10.1016/0169-5002(95)00450-F

[11] E. Saitoh, Y. Yokomizo, C. H. Cang, et al., "Cross-Cultural Validation of the Japanese Version of the Lung Cancer Subscale on the Functional Assessment of Cancer Therapy-Lung," Journal of Nippon Medical School, Vol. 74, No. 6, 2007, pp. 402-408. doi:10.1272/jnms.74.402

[12] M. Kenneth and Hargreaves, "Neuroendocrine Markers of Stress," Anesthesia Progress, Vol. 37, 1990, pp. 99-105.

[13] M. Biondi and A. Picardi, "Psychological Stress and Neuroendocrine Function in Humans: The Last Two Decades of Research," Psychotherapy and Psychosomatics, Vol. 68, No. 3, 1999, pp. 114-150. doi:10.1159/000012323

[14] C. Kirschbaum and D. H. Hellhammer, "Salivary Cortisol in Psychoneuroendocrine Research: Recent Developments and Applications," Psychoneuroendocrinology, Vol. 19, No. 4, 1994, pp. 313-333. doi:10.1016/0306-4530(94)90013-2

[15] Y. Noto, T. Sato, M. Kudo, et al., "The Relationship between Salivary Biomarkers and State-Trait Anxiety In- ventory Score under Mental Arithmetic Stress: A Pilot Study," Anesthesia \& Analgesia, Vol. 101, 2005, pp. 1873-1876. doi:10.1213/01.ANE.0000184196.60838.8D

[16] D. L. Delacroix and J. P. Vaerman, "Function of the Human Liver in IgA Homeostasis in Plasma," Annals of the New York Academy of Sciences, Vol. 409, No. 1, 1976, pp. 383-401. doi:10.1111/j.1749-6632.1983.tb26884.x

[17] H. Kubagawa, L. F. Bertoli, J. C. Barton, et al., "Analysis of Paraprotein Transport into the Saliva by Using AntiIdiotype Antibodies," Journal of Immunology, Vol. 138, 1987, p. 435.

[18] S. Tsujita and K. Morimoto, "Secretory IgA in Saliva Can Be Useful Stress Marker," Environmental Health and Preventive Medicine, Vol. 4, No. 1, 1999, pp. 1-8. doi:10.1007/BF02931243

[19] N. M. Graham, R. C. Bartholomeusz, N. Taboonpong, et al., "Dose Anxiety Reduce the Rate of Secretory IgA in Saliva?" Medical Journal of Australia, Vol. 148, No. 3, 1988, pp. 131-133.

[20] S. L. McDowell, R. A. Hughes, R. J. Hudhes, et al., "The Effect of Exercise Training on Salivary Immunoglobulin A and Cortisol Responses to Maximal Exercise," International Journal of Sports Medicine, Vol. 13, No. 8, 1992, pp. 577-580. doi:10.1055/s-2007-1024568

[21] D. Kajdaniuk, B. Marek, E. Swietochowska, et al., "Is Positive Correlation between Cortisol and Met-Enkephalin Concentration in Blood of Women with Breast Cancer a Reaction to Stress before Chemotherapy Administration?" Pathophysiology, Vol. 7, No. 1, 2000, pp. 47-51. doi:10.1016/S0928-4680(00)00028-6

[22] A. Montazeri, C. R. Gillis and J. McEwen, "Quality of life in Patients with Lung Cancer: A Review of Literature from 1970 to 1995," Chest, Vol. 113, No. 2, 1998, pp. 467-481. doi:10.1378/chest.113.2.467

[23] N. Lasikiewicza, H. Hendrickxb, D. Talbot, et al., "Exploration of Basal Diurnal Salivary Cortisol Profiles in Middle-Aged Adults: Associations with Sleep Quality and Metabolic Parameters," Psychoneuroendocrinology, Vol. 33, No. 2, 2008, pp. 143-151. doi:10.1016/j.psyneuen.2007.10.013

[24] J. M. Turner-Cobb, S. E. Sephton, C. Koopman, et al., "Social Support and Salivary Cortisol in Women with Metastatic Breast Cancer," Psychosomatic Medicine, Vol. 62, 2000, pp. 337-345. 